\title{
Integration vs. Segregation in Functional Brain Networks
}

\author{
I. Sendiña-Nadal, J.M. Buldú, I. Leyva, R. Bajo, J.A. Almendral, and F. del-Pozo,
}

\begin{abstract}
We propose a new methodology to evaluate the balance between segregation and integration in functional brain networks by using Singular Value Decomposition techniques. By means of magnetoencephalography, we obtain the brain activity of a control group of nineteen individuals during a memory task. Next, we project the node-to-node correlations into a complex network which is analyzed from the perspective of its modular structure encoded in the contribution matrix. In this way, we are able to study the role that nodes play inside/outside its community and to identify connector and local hubs. At the mesoscale level, the analysis of the contribution matrix allows to measure the degree of overlapping between communities and quantify how far the functional networks are from the configuration that better balances the integrated and segregated activity.
\end{abstract}

Index Terms-Functional brain networks, Complex Networks, Magnetoencephalography, Singular Value Decomposition.

\section{INTRODUCTION}

$\mathbf{F}$ ROM technological to biological systems, complex networks theory has been applied to a huge diversity of real data coming from the most different fields [1]. This methodology have also dealt with the brain, which is probably the most challenging system that we are facing in a biological context. The last years of studies have given us some hints about its anatomical structure [2], [3], [4], but we are still far from a complete knowledge. Studies in animal species such as C. Elegans [5], [6], cats or macaques [7], [8], have revealed common topological properties, such as high clustering and short topological distance between nodes, i.e., the fingerprint of a small-world (SW) network architecture [5]. With regard to the human brain, magnetic resonance imaging [2], [9] and diffusion spectrum imaging [4] have been used in order to obtain the pathways between cortical regions. Up to now, we know that the SW property is also present in the human brain together with exponential or truncated power-law decay in the degree distribution and the existence of certain communities inside the network [3], [4]. Several techniques as functional magnetic resonance imaging (fMRI), electroencephalography (EEG) and magnetoencephalography (MEG) have revealed the functional properties of the brain. These methods have shown that, although the anatomical structure is strongly correlated with the functional brain network in the resting state [10], very different functional networks arise depending on the task that the brain is performing [11]. There being important differences in the spatial and temporal resolution of these methods, all of them have shown the SW property also in the functional networks [12]. Small-worldness seems to play a crucial role in complex dynamical processes such as information transmission, pattern recognition or learning [13], but is not the only issue. Other studies have gone beyond the SW configuration and have quantified the importance of overconnected nodes [14] (known as hubs), unveiled the existence of characteristic network motifs [15], and also detected the appearance of community structures [16], which are related to the segregated organization of the brain.

In the present paper we are interested in how the existence of communities inside functional networks is related with the subtle balance between segregation and integration processes in the brain [17]. Traditionally, this problem has been treated in the context of anatomical networks and it has been related to the simultaneous presence of modules and their interconnections [18], [19]. Up to now, the analysis of modularity in functional brain networks have mainly focused in the detection of community structure or the characterization of the role played by the nodes inside their communities [16]. Nevertheless, less attention has been paid to the study of how the communities interact with each other due, in part, to the difficulty in evaluating the overlap between modules. Here, we apply a recently proposed method to evaluate the modular structure of complex networks by using Singular Value Decomposition [20]. The information given by this technique is twofold: on one hand, it allows to detect the role played by brain regions at the local and long-range scope, on the other, it quantifies the integration/segregation balance given by the functional communities and to evaluate how far it is from the optimal configuration. Although our sample study is based on magnetoencephalography (MEG) results obtained from a healthy control group during a memory task, the proposed method could be applied to any data set from healthy or impaired brain networks.

\section{METHODS}

\section{A. Subjects: Data Acquisition and Node-to-Node Correlations}

Nineteen right-handed, age-matched, healthy elderly volunteers, without memory complaints participated in the study. The group was chosen with an average number of eleven years of education. Individuals underwent a neuropsychological assessment, in order to establish their cognitive status in multiple cognitive functions. Next, a modified version of the Sternberg's letter-probe task [21] was used as the memory test. 
After memorizing a set of five letters, a series of single letters $(500 \mathrm{~ms}$ in duration with a random ISI between $2-3 \mathrm{~s}$ ) was presented, and the participants were asked to press a button with their right hand when a letter of the previous set was detected. During this task, the MEG signal was recorded with a $254 \mathrm{~Hz}$ sampling frequency and a band pass of 0.5 to $50 \mathrm{~Hz}$, using a 148-channel whole-head magnetometer. After applying a noise reduction algorithm, trials containing visible blinks, eye movements or muscular artifacts were excluded. Only hits (successful recognition of the letter) were considered since we were interested in evaluating the functional connectivity patterns which support recognition success. Next, we calculate the Synchronization Likelihood (SL) [22] between all pairs of nodes of each individual, being $N=148$ the total number of nodes (electrodes). Subsequently, SL was calculated for each of the thirty-five one-second epochs of the $(148 \times 147) / 2$ channel pairs, for the full-band signal (i.e., without bandfiltering), and for each subject. Finally, we obtained one $N \times N$ synchronization matrix $\mathbf{W}$ for each individual, where each component $w_{i j}$ was the average of the SL between nodes $i$ and $j$. Finally, all results were the average over the values obtained for each of the nineteen individuals.

\section{B. Projection of the modular structure}

We use the traditional partition into lobes as the community structure of the functional network. In this way, each node belongs to one of the $M=6$ brain lobes: Central (C), Frontal Left (FL), Frontal Right (FR), Temporal Left (TL), Temporal Right (TR) and Occipital (O). As proposed in [20] we use the Singular Value Decomposition (SVD) approach [23] in order to analyze the structure of the $N \times M$ contribution matrix C. The elements $C_{i \alpha}$ are the weights of the links of node $i$ that fall into community $\alpha, C_{i \alpha}=\sum_{j=1}^{N} w_{i j} S_{j \alpha}$, where $w_{i j}$ is the Synchronization Likelihood between nodes $i$ and $j$ and $\mathbf{S}$ is the partition matrix, where $S_{j \alpha}=1$ if the node $j$ belongs to community $\alpha$, and $S_{j \alpha}=0$ otherwise.

Next, we analyse $\mathbf{C}$ by using SVD [23] which consists on its factorization as $\mathbf{C}=\mathbf{U} \boldsymbol{\Sigma} \mathbf{V}^{\dagger}$, where $\mathbf{U}$ is a unitary $N \times N$ matrix and $\boldsymbol{\Sigma}$ is a diagonal $N \times M$ matrix whose elements are the singular values $\sigma_{i}$, which satisfy that $\sigma_{1}>\sigma_{2}>\ldots>\sigma_{M}$. Finally, $\mathbf{V}^{\dagger}$ is the conjugate transpose of the $M \times M$ unitary matrix V. By truncating the SVD we can obtain a least squares optimal reduced matrix $\mathbf{C}_{r}$ of order $r$ as $\mathbf{C}_{r}=\mathbf{U} \boldsymbol{\Sigma}_{r} \mathbf{V}^{\dagger}$. This can be done by considering only the $r$ highest values of $\sigma_{i}$ and resetting the others to zero. If we chose $r=2$ we are projecting all the information contained in the contribution matrix into a two dimensional space $\mathcal{U}_{2}$ formed by the two left singular vectors of matrix $\mathbf{U}$. In this space, it is easy to plot and analyze the projection of the contribution of nodes to a certain partition $\mathbf{n}_{i}=\sum_{\alpha=1}^{M} C_{i \alpha} \mathbf{e}_{\alpha}$, being $\mathbf{e}_{\alpha}=(0, \ldots, 0,1,0, \ldots 0)$ (a vector whose $\alpha$ th component is 1 and the rest are 0 ). We denote the projected contribution of the $i$ th node as $\tilde{\mathbf{n}}_{i}$ (see Fig. 1) and it is obtained as

$$
\tilde{\mathbf{n}}_{i}=\boldsymbol{\Sigma}_{2}^{-1} \mathbf{V}^{\dagger} \mathbf{n}_{i}
$$

where $\boldsymbol{\Sigma}_{2}^{-1}$ denotes the pseudo-inverse of the diagonal rectangular matrix $\boldsymbol{\Sigma}_{2}$, which only keeps the two largest singular values.

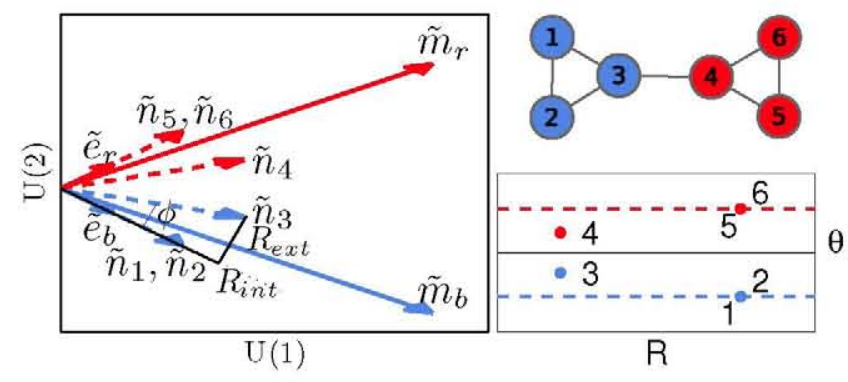

Fig. 1. Example scheme of the SVD $(r=2)$ for the top right network with two modules. (Left) The contribution of each node $\tilde{\mathbf{n}}_{i}$ is represented by a vector in the corresponding module color. All internal nodes, 1 and 2 in the blue module ( 5 and 6 in the red module), lie along the corresponding intramodular projection $\tilde{\mathbf{e}}_{b}\left(\tilde{\mathbf{e}}_{r}\right)$. The modular projection $\tilde{\mathbf{m}}_{b}\left(\tilde{\mathbf{m}}_{r}\right)$ is computed as the vector sum of all the $\tilde{\mathbf{n}}_{i}$ belonging to the blue (red) module. The relative distance of node 3 from its module is given by the angle $\phi$ and $\tilde{\mathbf{n}}_{3}$, which can be expressed as the sum of its components $R_{\text {int }}$ and $R_{\text {ext }}$. (Bottom right) Map of the node contributions to the intramodular projection directions in polar coordinates $R-\theta$, where $R_{i}$ is the norm of $\tilde{\mathbf{n}}_{i}$, and $\theta_{i}$ the angle between $\tilde{\mathbf{n}}_{i}$ and the horizontal axis. Dashed lines mark the directions of the intramodular projections of each module.

At the community level, we can calculate for each module $\alpha$ the line of the projections of its internal nodes (like the nodes 1 and 2 in Fig. 1). This direction $\tilde{\mathbf{e}}_{\alpha}$, called intramodular projection, gives the intrinsic direction that the community $\alpha$ has in the projection space $\mathcal{U}_{2}$ and it is obtained as:

$$
\tilde{\mathbf{e}}_{\alpha}=\boldsymbol{\Sigma}_{2}^{-1} \mathbf{V}^{\dagger} \mathbf{e}_{\alpha} .
$$

Note that, the contribution of each node in the projection space $\mathcal{U}_{2}$ can be expressed, using Eqs. (1) and (2), as a linear combination of intramodular projections, $\tilde{\mathbf{n}}_{i}=\sum_{\alpha=1}^{M} C_{i \alpha} \tilde{\mathbf{e}}_{\alpha}$.

Finally, every module $\alpha$ has a characteristic direction, the modular projection $\tilde{\mathbf{m}}_{\alpha}$, computed as the vector sum of all the projections of node contributions $\tilde{\mathbf{n}}_{i}$, for those nodes belonging to module $\alpha$, i.e.

$$
\overline{\mathbf{m}}_{\alpha}=\sum_{i=1}^{N} S_{i \alpha} \overline{\mathbf{n}}_{i} .
$$

Summarizing, the truncation of order two of the SVD leads to a vector associated to each node $\tilde{\mathbf{n}}_{i}$, and two vectors related with each community $\alpha$ : one that takes into account the contribution of all nodes of the community, $\overline{\mathbf{m}}_{\alpha}$, and other that only accounts for the internal weights $\tilde{\mathbf{e}}_{\alpha}$, the latest giving the intrinsic direction of the community $\alpha$. Figure 1 shows an schematic representation of the vectors $\tilde{\mathbf{n}}_{i}, \tilde{\mathbf{m}}_{\alpha}$ and $\tilde{\mathbf{e}}_{\alpha}$ for a test network. We can observe how every node with all links inside its community has a contribution vector $\overline{\mathbf{n}}_{i}$ in the direction of the intramodular community vector $\tilde{\mathbf{e}}_{\alpha}$. On the contrary, the more a node deviates from $\overline{\mathrm{e}}_{\alpha}$, the higher the participation in other communities.

\section{RESULTS}

\section{A. Community roles}

In order to unveil the role of the nodes in the structure of the modules (here taken as the brain lobes) of the recorded functional networks, we apply the proposed mapping (the loss of information associated with the $2 \mathrm{D}$ projection is $18.2 \%$ ) and 

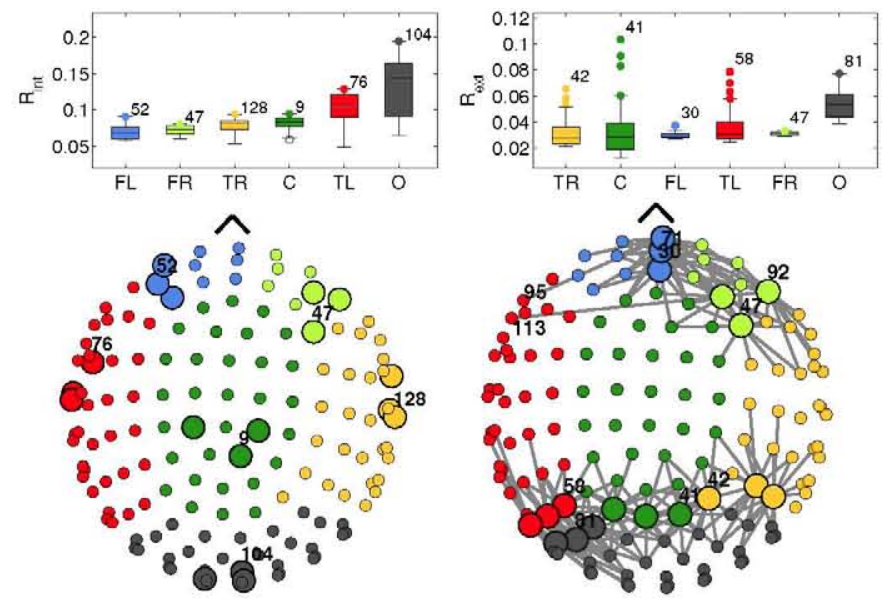

Fig. 2. (Top) Box-and-whisker plots of $R_{\text {int }}$ and $R_{\text {ext }}$. Lobes are sorted according to medians in increasing order. In each lobe, only outliers (defined as having a value more than 1.5 times the inter-quartile range IQR lower than the first quartile or 1.5 times IQR higher than the third quartile) and the node with the largest value are labeled. (Bottom) Outline of the group of 148 sensors overlying the cortex. Position of the three nodes with the highest internal contributions (left) and external contributions (right) are marked in each lobe. For the latter case, each highlighted node is connected to the 15 most synchronized neighbors.

calculate for each node $R_{\text {int }}=R \cos \phi$ and $R_{\text {ext }}=R \sin \phi$, being $\phi$ the absolute distance in angle between $\tilde{\mathbf{n}}_{\mathbf{i}}$ and the intramodular projection $\tilde{\mathrm{e}}_{\alpha}$ (see Fig. 1). While the distribution of $R_{\text {int }}$ for each module informs about the amount of the contribution of nodes comprising their own modules, $R_{\text {ext }}$ accounts for the heterogeneity in the connectivity with other lobes. In Fig. 2, we show the box-and-whisker plots of $R_{\text {int }}$ and $R_{\text {ext }}$ marking those nodes more capable to support the internal structure of the lobes (high $R_{\text {int }}$ ) and also to communicate them (high $R_{\text {ext }}$ ). We observe that, in this optimal mapping for $R_{i n t}$, all lobes have similar medians, while the occipital lobe has a median larger than the percentiles-75 of the rest, indicating that this lobe is highly functional cohesive. Regarding the interlobe communication, the similarity in range and medians reveals the homogeneity of the mesoscale, being the occipital lobe the one with highest value. Nevertheless, the role played by the two temporal and central lobes is still very significant because of their high outliers. In the bottom plots of Fig. 2 we show the position of the local hubs, i.e. those nodes with higher $R_{\text {int }}$ at their lobes, and the connector hubs, which are those nodes with higher $R_{\text {ext }}$.

\section{B. Integration vs. Segregation}

Next, we are concerned about the mesoscopic interactions inside the network, i.e., how lobes overlap with each other and their implications in the integrated/segregated activity of the functional network. With this aim, we measure the segregated activity of all modules as $M_{S}=\frac{1}{M} \sum_{\alpha} \frac{\tilde{\mathbf{e}}_{\alpha}}{\left\|\tilde{\mathbf{e}}_{\alpha}\right\|} \frac{\tilde{\mathbf{n}}_{\alpha}}{\left\|\tilde{\mathbf{m}}_{\alpha}\right\|}$. Note that the lower the connectivity of a lobe $\alpha$ with the rest of the lobes, the closer the vectors $\overline{\mathrm{e}}_{\alpha}$ and $\overline{\mathbf{m}}_{\alpha}$, leading to a value of $M_{S \alpha}$ close to one. In the absence of interlobe connections, $\tilde{\mathbf{e}}_{\alpha}=\tilde{\mathbf{m}}_{\alpha}$ and $M_{S \alpha}=1$. At the same time, we measure the overlap between lobes as their difference in their community vectors. The indicator of the modular integration is obtained as
$M_{I}=\frac{1}{M(M-1)} \sum_{\alpha \neq \beta} \frac{\tilde{\mathbf{m}}_{\alpha}}{\left\|\tilde{\mathbf{m}}_{\alpha}\right\|} \frac{\tilde{\mathbf{m}}_{\beta}}{\left\|\tilde{\mathbf{m}}_{\beta}\right\|}$. Finally, we introduce the modular balance $M_{B}$ as the product of the modular integration and the modular segregation $M_{B}=M_{I} \cdot M_{S}$. To understand how optimal is the real configuration of the functional brain networks we tune (by multiplying) the weight of the interlobe connections by a parameter $\mu$. In this way, when $\mu=0$ all interlobe connections are deleted, giving rise to a network broken into six lobes. When $\mu=1$, we recover the real values of the interlobe connections, while for $\mu=2$ the interlobe weights are doubled. In Fig. 3 we show the variation of $M_{I}, M_{S}$ and $M_{B}$ as a function $\frac{\langle w\rangle_{\text {out }}}{\langle w\rangle_{\text {in }}}$, being $\langle w\rangle_{\text {in }}$ and $\langle w\rangle_{\text {out }}$ the average weight of the intralobe and interlobe links, respectively, the latter depending on the value of $\mu$. From now on, all parameters are calculated for $r=6$. We observe that modular segregation $M_{S}$ (blue circles) is a monotonous decreasing function while the modular integration $M_{I}$ (red squares) increases from 0 to a constant value. Interestingly, there exists an optimal balance between these two quantities as reflected by the maximum present in the modular balance $M_{B}$ (black triangles). The dashed line of Fig. 3 shows the value of the modular quantities for $\mu=1$, i.e., the real values of the functional networks. It is clear that the real modular balance $M_{B}$ is close, but not equal, to the maximum observed when interlobe connections are modified. This optimal configuration, which maximizes the balance between segregation and integration, is obtained for $\mu=1.8$, indicating that a further increase of the interlobe connections would lead to a more balanced structural configuration.

Finally, in Fig. 4 we plot the overlap matrix $\mathrm{O}$ between lobe regions, whose components are obtained from the scalar product of the modular projections $O_{\alpha \beta}=\frac{\overline{\mathbf{m}}_{\alpha}}{\left\|\tilde{\mathbf{m}}_{\alpha}\right\|} \frac{\tilde{\mathbf{m}}_{\beta}}{\left\|\tilde{\mathbf{m}}_{\beta}\right\|}$. In the real functional networks we observe a maximum overlap between the frontal left (FL) and frontal right (FR) lobes, followed by the overlap of the Central (C) lobe with the FL (top left panel). Interestingly, the FL-FR and C-FL overlap are also the ones with higher values in the optimal configuration (top right panel). Nevertheless, this overlap structure is lost both when decreasing (bottom left) or increasing (bottom right) the strength of the interlobe connections. Despite not

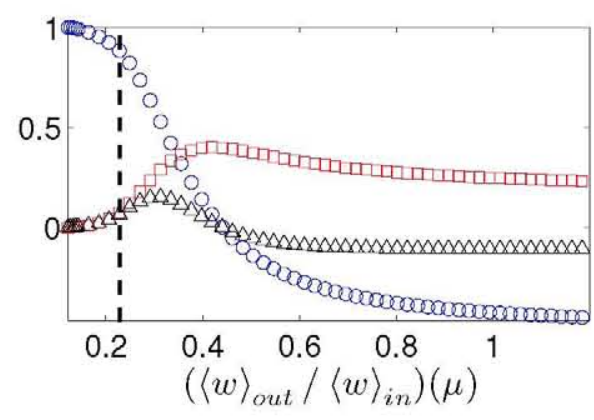

Fig. 3. Segregation and integration balance as a function of the $\mu$ dependent ratio between the external and internal weights per link. Blue circles represent the modular segregation $M_{S}$. Red squares provide the modular integration $M_{I}$ and black triangles are the modular balance $M_{B}$, the product of both quantities. Dashed line indicates the value in the real functional networks $(\mu=1)$. There is an optimal ratio between internal and external weights for which the balance between integration and segregation is maximal ( $\mu=1.8$ ). 


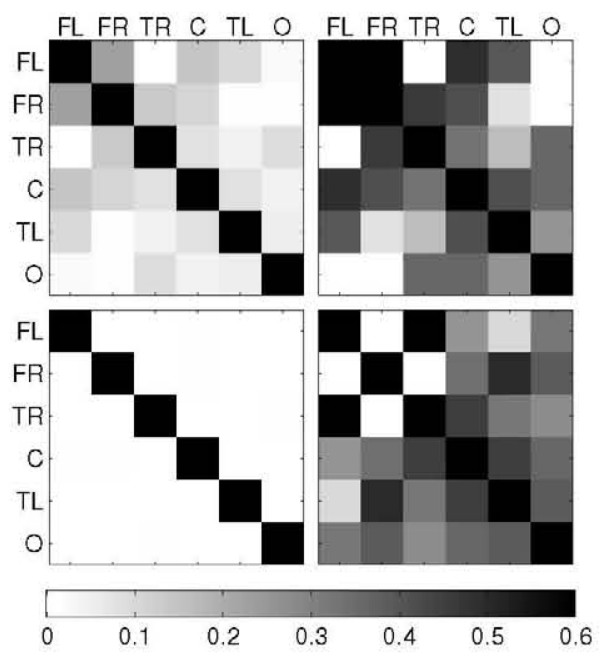

Fig. 4. Overlap matrices $O$ between lobes. Each matrix corresponds to the normalized scalar product of the individual modular projections $\tilde{\mathbf{m}}_{\alpha}$ obtained by tuning the weight of the external links with a factor $\mu$. From top to bottom and from left to right: $\mu=1$ (real network), $\mu=1.8$ (optimal value of $M_{B}$ ), $\mu=0.2$ (low interlobe connectivity) and $\mu=5$ (high interlobe connectivity).

being optimal, those regions with higher overlap in the real functional networks coincide with those predicted by the optimal configuration.

\section{CONCLUSIONS}

We have used the Singular Value Decomposition for the analysis of the modular structure of functional brain networks obtained by magnetoencephalography during a memory task. With this method, we have quantified the contribution of brain areas to the intralobe/interlobe activity and we have detected those regions having the leading role inside each lobe and those being the main channels of interlobe communication. Next, we have measured the amount of overlap between lobes, and we have studied how the variation of the interlobe communication would modify the balance between segregation and integration. Finally, we have seen that the modular structure of the functional networks analyzed here is close to the optimal configuration. We believe that this kind of analysis could be applied to further works comparing healthy individuals with patients suffering from different brain diseases.

\section{ACKNOWLEDGMENT}

This work was supported by the Spanish Ministry of S\&T [FIS2009-07072] and by the Community of Madrid under project URJC-CM-2010-CET-5006 and the R\&D Program of activities MODELICO-CM [S2009ESP-1691].

\section{REFERENCES}

[1] Boccaletti, S., Latora, V., Moreno, Y., Chavez, M., and Hwang, D.-U., "Complex Networks: Structure and Dynamics", Physics Reports, 424, 175 (2006).

[2] He, Y., Chen, Z.J. and Evans, A.C., "Small-world anatomical networks in the human brain revealed by cortical thickness from MRI", Cerebral Cortex 17, 2407-2419 (2007).

[3] Hangmann, P., Kurant, M., Gigandet, X., Thiran, P., Wedeen, V.J., Meuli, R., and Thiran, J.P., "Mapping human whole-brain structural networks with diffusion MRI", PLOS ONE, 7, e597 (2007).
[4] Hagmann, P., Cammoun, L., Gigandet, X., Meuli, R., Honey, C.J., Wedeen, V.J. and Sporns, O., "Mapping the Structural Core of Human Cerebral Cortex", PLoS Biology, 6, e159 (2008).

[5] Watts, D.J. and Strogatz, S.H., "Collective dynamics of small-world networks", Nature, 393, 440 (1998)

[6] Kaiser, M. and Hiltetag, C.C., "Nonoptimal Component Placement, but Short Processing Paths, due to Long-Distance Projections in Neural Systems", PLoS Comput Biol., 2, e95 (2006).

[7] Hilgetag, C.C., Burns, G.A.P.C., O'Neill, M.A.O., Scannell, J.W. and Young, M.P., "Anatomical connectivity defines the organization of clusters of cortical areas in the macaque monkey and the cat", Phil. Trans. $R$. Soc. Lond. B, 355, 91-110 (2000).

[8] Hilgetag, C.C. and Kaiser, M., "Clustered organization of cortical connectivity", Neuroinformatics, 2 353-360 (2004).

[9] Iturria-Medina, Y., Canales-Rodríguez, E.J., Melie-García, L., ValdésHernández, P.A., Martínez-Montes, E., Alemán-Gómez, Y., and SánchezBornot J.M., "Characterizing brain anatomical connections using diffusion weighted MRI and graph theory", Neuroimage, 36, 645-660 (2007).

[10] Achard, S., Salvador, R., Whitcher, B., Suckling, J. and Bullmore, E., "A resilient low-frequency, small-world human brain functional network with highly connected association cortical hubs", J. Neurosci.,26, 63 (2006).

[11] Schinkel, S., Zamora-López, G., Dimingen O., Sommer, W. and Kurths, J., "Functional network analysis reveals differences in the semantic priming taks", J. Neurosc. Methods 97, 333-339 (2011).

[12] Bullmore, K. and Sporns, O., "Complex brain networks: graph theoretical analysis of structural and functional systems", Nature, 10, 186 (2009).

[13] Simard, D., Nadeau, L., and Kröger, H., "Fastest learning in small-world neural networks", Phys. Lett. A, 336, 8-15 (2005).

[14] Sporns, O., Tononi, G. and Kötter, R., "Identification and classification of hubs in brain networks", PLoS ONE, 2, e1049 (2007).

[15] Sporns, O., and Kötter, R., "Motifs in brain networks", PLoS Biol., 2, 1910-1918 (2004)

[16] Meunier, D., Lambiotte, R., and Bullmore, E., "Modular and hierarchically modular organization of brain networks", Frontiers in Neurocience, 4, 200(1-11) (2010).

[17] Tononi, G., Sporns, O. and Edelman, G.M., "A measure for brain complexity: Relating functional segregation and integration in the nervous system", Proc. Natl. Acad. Sci. USA, 91, 5033-5037 (1994).

[18] Tononi, G., Edelman, G.M., and Sporns, O., "Complexity and coherency: integrating information in the brain", Trends Cogn. Sci. 2, 474 (1998).

[19] Sporns, O., and Tononi, G., "Classes of network connectivity and dynamics", Complexity, 7, 28-38 (2001).

[20] Arenas, A., Borge-Holthoefer, J., Gómez S., and Zamora-Lopez, G., "Optimal map of the modular structure of complex networks", New. I. Phys, 12, 053009 (2010).

[21] Maestu, F., Fernandez, A., Simos, P.G., Gil-Gregorio, P., Amo, C., Rodriguez, R., Arrazola, J., Ortiz, T., "Spatio-temporal patterns of brain magnetic activity during a memory task in Alzheimer's disease", Neuroreport, 12, 3917-3922 (2001).

[22] Stam, C.J. and van Dijk, B.W., "Synchronization likelihood: an unbiased measure of generalized synchronization in multivariate data sets", Physica D, 163, 236-251 (2002)

[23] Golub G.H. and Van Loan, C.F., "Matrix Computations", 3rd edn (Baltimore: Johns Hopkins University Press), 1996.

I. Sendiña-Nadal is an Assoc. Prof. at the Univ. Rey Juan Carlos (URJC) and member of the Biological Networks Lab. (Biomedical Technology Center, Spain). She works on synchronization in Biological Networks.

Javier M. Buldú is an Assoc. Prof. at the URJC and member of the Biological Networks Lab. (Biomedical Technology Center, Spain). He works on the dynamics of nonlinear systems and applications of Complex Networks Theory.

I. Leyva is an Associate Prof. at URJC and member of the Biological Networks Lab. (Biomedical Technology Center, Spain). She works on the synchronization of nonlinear systems in the context of Complex Networks.

R. Bajo is at the MEG Centre of the Medicine Faculty (UCM) and at the Cognitive and Computational Neuroscience Lab. (Biomedical Technology Center, Spain). He works on the application of techniques from nonlinear systems and signal processing to MEG and EEG data.

J. A. Almendral is an Associate Prof. at URJC and member of the Biological Networks Lab. at the Centre for Biomedical Technology (Spain). His research is focused on structure/function relationship in collective phenomena.

F. del-Pozo is Full Prof. at the UPM and the Director of the Bioengineering and Telemedicine Group and the Biomedical Technology Centre of UPM. Head of the Bioengineering and Medical Imaging area of the CIBER-bbn and the Bioscience Madrid Research Program on Biomedical Engineering. 